\title{
Ultrafiltration in Decompensated Heart Failure with Cardiorenal Syndrome
}

\author{
Bradley A. Bart, M.D., Steven R. Goldsmith, M.D., Kerry L. Lee, Ph.D., Michael M. Givertz, \\ M.D., Christopher M. O'Connor, M.D., David A. Bull, M.D., Margaret M. Redfield, M.D., Anita \\ Deswal, M.D., M.P.H., Jean L. Rouleau, M.D., Martin M. LeWinter, M.D., Elizabeth O. Ofili, \\ M.D., M.P.H., Lynne W. Stevenson, M.D., Marc J. Semigran, M.D., G. Michael Felker, M.D., \\ Horng H. Chen, M.D., Adrian F. Hernandez, M.D., Kevin J. Anstrom, Ph.D., Steven E. \\ McNulty, M.S., Eric J. Velazquez, M.D., Jenny C. Ibarra, R.N., M.S.N., Alice M. Mascette, \\ M.D., and Eugene Braunwald, M.D. for the Heart Failure Clinical Research Network \\ Hennepin County Medical Center, Minneapolis (B.A.B., S.R.G.) and Mayo Clinic, Rochester \\ (M.M.R., H.H.C.) - both in Minnesota; Duke Clinical Research Institute (K.L.L., A.F.H., K.J.A., \\ S.E.M., E.J.V., J.C.I.) and Duke University Medical Center and Duke Heart Center (C.M.O., \\ G.M.F.) - both in Durham, NC; Brigham and Women's Hospital (M.M.G., L.W.S., E.B.) and \\ Massachusetts General Hospital (M.J.S.) - both in Boston; University of Utah, Salt Lake City \\ (D.A.B.); Michael E. DeBakey VA Medical Center and Baylor College of Medicine, Houston \\ (A.D.); University of Montreal and Montreal Heart Institute, Montreal (J.L.R.); University of \\ Vermont, Burlington (M.M.L.); Morehouse School of Medicine, Atlanta (E.O.O.); and the National \\ Heart, Lung, and Blood Institute, Bethesda, MD (A.M.M.)
}

\footnotetext{
Abstract

BACKGROUND-Ultrafiltration is an alternative strategy to diuretic therapy for the treatment of patients with acute decompensated heart failure. Little is known about the efficacy and safety of ultrafiltration in patients with acute decompensated heart failure complicated by persistent congestion and worsened renal function.

METHODS-We randomly assigned a total of 188 patients with acute decompensated heart failure, worsened renal function, and persistent congestion to a strategy of stepped pharmacologic therapy (94 patients) or ultrafiltration ( 94 patients). The primary end point was the bivariate change from baseline in the serum creatinine level and body weight, as assessed 96 hours after random assignment. Patients were followed for 60 days.

RESULTS-Ultrafiltration was inferior to pharmacologic therapy with respect to the bivariate end point of the change in the serum creatinine level and body weight 96 hours after enrollment $(\mathrm{P}=0.003)$, owing primarily to an increase in the creatinine level in the ultrafiltration group. At 96 hours, the mean change in the creatinine level was $-0.04 \pm 0.53 \mathrm{mg}$ per deciliter $(-3.5 \pm 46.9 \mu \mathrm{mol}$ per liter) in the pharmacologic-therapy group, as compared with $+0.23 \pm 0.70 \mathrm{mg}$ per deciliter $(20.3 \pm 61.9 \mu \mathrm{mol}$ per liter) in the ultrafiltration group $(\mathrm{P}=0.003)$. There was no significant difference in weight loss 96 hours after enrollment between patients in the pharmacologic-therapy group and those in the ultrafiltration group (a loss of $5.5 \pm 5.1 \mathrm{~kg}[12.1 \pm 11.3 \mathrm{lb}]$ and $5.7 \pm 3.9 \mathrm{~kg}$ [12.6 $\pm 8.5 \mathrm{lb}$ ], respectively; $\mathrm{P}=0.58)$. A higher percentage of patients in the ultrafiltration group than in the pharmacologic-therapy group had a serious adverse event ( $72 \%$ vs. $57 \%, \mathrm{P}=0.03)$.
}

Copyright (C) 2012 Massachusetts Medical Society.

Address reprint requests to Dr. Bart at O5 HCMC, 701 Park Ave. South, Minneapolis, MN 55410, or at bartx006@umn.edu. Disclosure forms provided by the authors are provided with the full text of this article at NEJM.org. 
CONCLUSIONS-In a randomized trial involving patients hospitalized for acute decompensated heart failure, worsened renal function, and persistent congestion, the use of a stepped pharmacologic-therapy algorithm was superior to a strategy of ultrafiltration for the preservation of renal function at 96 hours, with a similar amount of weight loss with the two approaches. Ultrafiltration was associated with a higher rate of adverse events.

The acute cardiorenal syndrome (type 1) is defined as worsening renal function in patients with acute decompensated heart failure. ${ }^{1}$ It occurs in 25 to $33 \%$ of patients with acute decompensated heart failure and is associated with poor outcomes. ${ }^{1,2}$ Multiple processes contribute to the development of the acute cardiorenal syndrome, including extrarenal hemodynamic changes, neurohormonal activation, intrarenal microvascular and cellular dysregulation, and oxidative stress. ${ }^{1}$ In some cases, intravenous diuretics, which are often administered in patients with acute decompensated heart failure, ${ }^{3}$ may directly contribute to worsening renal function. ${ }^{1,4,5}$ The use of diuretics to treat persistent congestion after the onset of worsening renal function may lead to further kidney injury.

Venovenous ultrafiltration is an alternative therapy in this setting. Potential advantages of ultrafiltration include greater control over the rate and volume of fluid removal, greater net loss of sodium, and less neurohormonal activation. ${ }^{6}$ Current treatment guidelines state that ultrafiltration is a reasonable approach in patients with congestion that is not responding to medical therapy (class IIa, level of evidence B). ${ }^{3}$ However, little is known about the safety and efficacy of ultrafiltration as compared with pharmacologic therapy in patients with acute decompensated heart failure complicated by acute cardiorenal syndrome and persistent congestion. ${ }^{4}$ Therefore, we designed the Cardiorenal Rescue Study in Acute Decompensated Heart Failure (CARRESS-HF) to compare the effect of ultrafiltration with that of stepped pharmacologic therapy on renal function and weight loss in patients with heart failure who have worsening renal function and persistent congestion. ${ }^{7}$

\section{METHODS \\ STUDY OVERSIGHT}

The National Heart, Lung, and Blood Institute (NHLBI)-sponsored Heart Failure Network conceived, designed, and conducted the CARRESS-HF. The trial protocol was approved by a protocol review committee and a data and safety monitoring board, both appointed by the NHLBI, and by the institutional review board at each participating site. All study-related activities, including the collection and analysis of the data, were coordinated by the data coordinating center at the Duke Clinical Research Institute. The first draft of the manuscript was written by the first author, and the final draft was revised, reviewed, and approved by all the authors. All the authors assume responsibility for the overall content and integrity of the article. The authors, steering committee, and executive committee of the Heart Failure Network made the decision to submit the manuscript for publication and vouch for the data and analysis and for the fidelity of this report to the study protocol (which is available with the full text of this article at NEJM.org). CHF Solutions (Brooklyn Park, MN) provided limited financial support for the purchase of ultrafiltration filters but had no role in the conduct of the trial, analysis of the data, or interpretation of the results. No data or draft of the manuscript was shared with CHF Solutions before publication.

\section{STUDY DESIGN}

The design of and rationale for the trial have been described previously. ${ }^{7}$ The CARRESS$\mathrm{HF}$ was a randomized trial that compared ultrafiltration with a strategy of diuretic-based stepped pharmacologic therapy. Patients who were hospitalized with acute decompensated heart failure as the primary diagnosis were eligible for enrollment. There was no exclusion 
criterion that was based on ejection fraction. All patients had worsened renal function (defined as an increase in the serum creatinine level of at least $0.3 \mathrm{mg}$ per deciliter [26.5 $\mu$ mol per liter]) within 12 weeks before or 10 days after the index admission for heart failure. All patients were required to have at least two of the following conditions at the time of randomization: at least 2+ peripheral edema, jugular venous pressure greater than $10 \mathrm{~cm}$ of water, or pulmonary edema or pleural effusion on chest radiography. Patients with a serum creatinine level of more than $3.5 \mathrm{mg}$ per deciliter (309.4 $\mu \mathrm{mol}$ per liter) at the time of admission and those receiving intravenous vasodilators or inotropic agents were excluded from the study. A complete list of the trial inclusion and exclusion criteria is provided in the Supplementary Appendix, available at NEJM.org.

All study participants provided written informed consent before randomization. With the use of an automated Web-based system, patients were randomly assigned, in a 1:1 ratio, to either ultrafiltration therapy or pharmacologic therapy. A permuted-block randomization scheme was used, with stratification according to clinical site.

For patients assigned to ultrafiltration therapy, loop diuretics were to be discontinued for the duration of the ultrafiltration intervention. Fluid status was managed by means of ultrafiltration with the use of the Aquadex System 100 (CHF Solutions) according to the manufacturer's specifications. Ultrafiltration was performed at a fluid-removal rate of 200 $\mathrm{ml}$ per hour. The addition of intravenous vasodilators or positive inotropic agents after randomization was prohibited unless they were deemed to be necessary as rescue therapy.

For patients assigned to stepped pharmacologic therapy, intravenous diuretics were used to manage signs and symptoms of congestion. Investigators were encouraged to decrease doses, increase doses, or continue current doses of diuretics as necessary to maintain a urine output of 3 to 5 liters per day. Recommendations regarding the use of intravenous vasodilators and inotropic agents for patients in whom the target urine output could not be attained were based on the individual patient's blood pressure, ejection fraction, and the presence or absence of right ventricular failure at 48 hours. The details of the stepped pharmacologic-therapy algorithm are provided in the Supplementary Appendix.

In both groups, the assigned treatment strategy was to be continued until the signs and symptoms of congestion in the patient were reduced to the best extent possible. Crossover was discouraged. Diuresis or ultrafiltration could be slowed or temporarily discontinued to address technical problems or clinical care requirements, as determined by the treating physician.

\section{TRIAL END POINTS}

The primary end point was the change in the serum creatinine level and the change in weight, considered as a bivariate response, between the time of randomization and 96 hours after randomization. ${ }^{7}$ The use of a bivariate primary end point (in which the change in serum creatinine level and the change in weight are considered simultaneously, with the results displayed on a two-dimensional grid) was intended to allow for the integration of two clinically important outcomes. ${ }^{8}$ Secondary end points included the rate of clinical decongestion and measures of global well-being and dyspnea. Clinical decongestion was defined as jugular venous pressure of less than $8 \mathrm{~cm}$ of water, no more than trace peripheral edema, and the absence of orthopnea. Global well-being and dyspnea were assessed with the use of a visual-analogue scale that ranged from 0 to 100, with higher scores indicating greater well-being and less severity of dyspnea, respectively. ${ }^{9}$ A complete listing of secondary end points is provided in the Supplementary Appendix. 


\section{STATISTICAL ANALYSIS}

On the basis of data from the Ultrafiltration versus Intravenous Diuretics for Patients Hospitalized for Acute Decompensated Congestive Heart Failure (UNLOAD) trial, ${ }^{10}$ the standard deviation of the change in weight at 96 hours was estimated to be 3.1 to $3.5 \mathrm{~kg}$ ( 6.8 to $7.7 \mathrm{lb}$ ), and the standard deviation of the change in the creatinine level at 96 hours was estimated to be 0.55 to $0.75 \mathrm{mg}$ per deciliter ( 48.6 to $66.3 \mu \mathrm{mol}$ per liter). We estimated that with enrollment of 200 participants, the study would have more than $90 \%$ power to detect a difference of $0.5 \mathrm{SD}$ between the treatment groups for each component of the bivariate primary end point.

The primary analyses were based on the intention-to-treat principle. Baseline characteristics are presented as means and standard deviations or medians and interquartile ranges. The response of each patient with respect to the bivariate primary end point was displayed on a two-dimensional grid representing changes in the creatinine level and changes in weight 96 hours after randomization. For the comparison of treatment groups in the primary analysis, we used a multivariate linear-regression model, adjusting for baseline weight and creatinine level. ${ }^{7}$ A 95\% confidence region (an ellipse) for the mean bivariate response (change in creatinine level and change in weight) in each treatment group and for the average betweengroup difference in the bivariate response was identified with the use of the multivariate linear-regression model framework. ${ }^{8,11}$

The primary analysis was based on results of creatinine testing performed at the core laboratory, when those were available; otherwise, the results of testing performed at local laboratories were used. Body weight was measured by research personnel with the use of a locally available scale. Site personnel were encouraged to use the same scale for all patients whenever possible (and otherwise, the same scale for all weight assessments of a particular patient) and to weigh patients in the morning before breakfast, with patients wearing hospital gowns and no shoes. In the case of patients for whom 96-hour data were missing owing to death or early discharge from the hospital (13 patients in each treatment group), the last-observation-carried-forward method was used for imputation of data on creatinine level and weight. Two patients who were randomly assigned to ultrafiltration were not included in the primary end-point analysis owing to missing baseline data on creatinine level (1 patient) or lack of all post-baseline data on creatinine level ( 1 patient). Cumulative event rates for secondary end points involving time-to-event data were estimated with the use of the Kaplan-Meier method. ${ }^{12}$ Hazard ratios, their $95 \%$ confidence intervals, and $\mathrm{P}$ values for the comparison of the two treatment groups were determined with the use of the Cox regression model. ${ }^{13} \mathrm{~A}$ two-sided alpha level of 0.05 was considered to indicate statistical significance. All data analyses were conducted with the use of SAS software, version 9.2.

\section{RESULTS PATIENTS}

Patients were enrolled in the trial between June 22, 2008, and January 27, 2012, at 22 sites in the United States and Canada. Enrollment ended on February 3, 2012, on the recommendation of the data and safety monitoring board, after 188 of the planned 200 patients had been enrolled, because of a lack of evidence of benefit, as well as an excess of adverse events, with ultrafiltration.

A total of 94 patients were enrolled in each treatment group. The baseline demographic and clinical characteristics are shown in Table 1 . The median age of the population was 68 years, $75 \%$ of the patients were men, $85 \%$ had hypertension, and $66 \%$ had diabetes mellitus. The median ejection fraction was $33 \%$. A total of $77 \%$ of the patients had been hospitalized for heart failure during the previous year. The median time from the index hospital admission 
(the admission qualifying the patient for enrollment in the study) to random assignment to a treatment group was 34 hours. The qualifying serum creatinine value was obtained after admission to the hospital in $95 \%$ of the participants. The median qualifying increase in the creatinine level was $0.45 \mathrm{mg}$ per deciliter (39.8 $\mu \mathrm{mol}$ per liter).

\section{STUDY TREATMENTS}

All 94 patients in the pharmacologic-therapy group received intravenous diuretics. The median duration of the stepped pharmacologic-therapy intervention was 92 hours (interquartile range, 56 to 138). The primary reasons that stepped pharmacologic therapy was discontinued were the following: the best possible fluid volume was reached (72\% of the patients), the creatinine level was increased (12\%), there was evidence of intravascular volume depletion (3\%), and blood pressure dropped or clinical instability developed (2\%). Six participants $(6 \%)$ in the pharmacologic-therapy group underwent ultrafiltration during the first 7 days (two of whom underwent ultrafiltration before the primary end-point assessment on day 4). In addition to receiving loop diuretics, $46 \%$ of the participants in the pharmacologic-therapy group received treatment with metolazone within the first 7 days, $5 \%$ were treated with intravenous vasodilators, and $12 \%$ were treated with intravenous inotropic agents before the day 4 assessment.

Ultrafiltration was started a median of 8 hours after random assignment, and the median duration of the treatment was 40 hours (interquartile range, 28 to 67). The primary reasons that ultrafiltration was stopped were the following: the best possible fluid volume was reached (50\% of the patients), the creatinine level was increased (16\%), difficulties developed with vascular access (9\%), and thrombosis of the ultrafiltration circuit developed (9\%). Eight patients (9\%) in the ultrafiltration group received intravenous diuretics instead of ultrafiltration, and an additional $28(30 \%)$ received intravenous diuretics after ultrafiltration was stopped and before the 96-hour assessment. A total of 3\% of the patients received vasodilators and 3\% received intravenous inotropic agents before the day 4 assessment. Randomization, treatment, and follow-up of the patients are shown in Figure S1 in the Supplementary Appendix.

\section{PRIMARY END POINT}

There was a significant difference between the treatment groups in the bivariate end point of change in weight and change in serum creatinine level 96 hours after enrollment $(\mathrm{P}=0.003)$ (Fig. 1, and Fig. S2 in the Supplementary Appendix). This difference was due primarily to an increase in the serum creatinine level in the ultrafiltration group. At 96 hours, the mean change in the serum creatinine level from the level measured at the time of randomization was a decrease of $0.04 \pm 0.53 \mathrm{mg}$ per deciliter $(3.5 \pm 46.9 \mu \mathrm{mol}$ per liter) in the pharmacologic-therapy group, as compared with an increase of $0.23 \pm 0.70 \mathrm{mg}$ per deciliter $(20.3 \pm 61.9 \mu$ mol per liter) in the ultrafiltration group ( $\mathrm{P}=0.003)$. There was no significant difference between pharmacologic therapy and ultrafiltration with respect to the mean weight loss 96 hours after enrollment $(5.5 \pm 5.1 \mathrm{~kg}[12.1 \pm 11.3 \mathrm{lb}]$ and $5.7 \pm 3.9 \mathrm{~kg}[12.6 \pm 8.5$ lb] in the two groups, respectively; $\mathrm{P}=0.58$ ).

\section{SECONDARY END POINTS}

The changes from baseline in the creatinine level at 48, 72, and 96 hours and at 60 days differed significantly between the patients in the pharmacologic-therapy group and those in the ultrafiltration group (Fig. 2A). However, there were no significant differences between the treatment groups at the time of discharge or on day 7, whichever occurred first, or at the 30-day assessment. There were no significant between-group differences in weight at any of the time points (Fig. 2B). 
The rate of clinical decongestion at 96 hours was low in the two treatment groups (9\% with pharmacologic therapy and $10 \%$ with ultrafiltration, $\mathrm{P}=0.83$ ) (Table 2). Within the first 7 days, there was no significant difference between the groups in the percentage of participants whose condition worsened (with worsening condition defined as death, worsening or persistent heart failure, need for dialysis, or the occurrence of a serious adverse event) or who crossed over to alternate therapy (18\% with pharmacologic therapy and $23 \%$ with ultrafiltration, $\mathrm{P}=0.45$ ) or the change in the furosemide-equivalent dose of diuretics (an increase of $2.2 \mathrm{mg}$ per day in the pharmacologic-therapy group and a decrease of $20.6 \mathrm{mg}$ per day in the ultrafiltration group, $\mathrm{P}=0.18$ ). At 96 hours and at day 7 or hospital discharge, there were no significant between-group differences in scores on the dyspnea and global well-being visual-analogue scales (Table 2). The total fluid output differed significantly between the two groups on the second day after randomization but not on days 1, 3, or 4 (Fig. S3 in the Supplementary Appendix).

\section{SERIOUS ADVERSE EVENTS AND CLINICAL OUTCOMES}

A higher percentage of patients in the ultrafiltration group than in the pharmacologictherapy group had a serious adverse event over the 60-day period of follow-up (72\% vs. $57 \%, \mathrm{P}=0.03$ ). The higher percentage in the ultrafiltration group was attributable mainly to higher incidences of kidney failure, bleeding complications, and intravenous catheter-related complications (Table 3).

The 60-day estimated mortality was $17 \%$ in the ultrafiltration group, as compared with $13 \%$ in the pharmacologic-therapy group ( $\mathrm{P}=0.47$ ) (Fig. S4 in the Supplementary Appendix). There was no significant difference in the composite rate of death or rehospitalization for heart failure (38\% and 35\%, respectively; $\mathrm{P}=0.96$ ) (Fig. S5 in the Supplementary Appendix) or in the composite rate of death or rehospitalization for any reason (61\% and 48\%, respectively; $\mathrm{P}=0.12$ ) (Fig. S6 in the Supplementary Appendix).

\section{DISCUSSION}

In CARRESS-HF, we compared ultrafiltration with diuretic-based therapy in patients with acute decompensated heart failure and worsened renal function. The serum creatinine level 96 hours after enrollment was significantly increased in the ultrafiltration group as compared with the pharmacologic-therapy group but the weight loss was not significantly greater. There were also no significant between-group differences in weight loss, mortality, or the rate of hospitalization for heart failure during the 60-day follow-up period. Given the high cost and complexity of ultrafiltration, the use of this technique as performed in the current study does not seem justified for patients hospitalized for acute decompensated heart failure, worsened renal function, and persistent congestion.

The reason for the early rise in the creatinine level in the patients who underwent ultrafiltration is unclear. It is possible that these patients had transient intravascular volume depletion during ultrafiltration. Previous studies examining plasma refill rates in patients with heart failure have shown that rates of volume removal greater than $200 \mathrm{ml}$ per hour, which was the rate used in our trial, are not associated with adverse effects. ${ }^{14}$ The duration of ultrafiltration was longer in the present study than it was in other trials, ${ }^{10,14,15}$ a fact that may have contributed to the increase in the creatinine level at 96 hours in the patients who received this treatment. At 30 days and at 60 days, the mean creatinine level was below the baseline level in both treatment groups. The lesser reduction in the creatinine level in the ultrafiltration group may be real, but it may also represent a chance finding or an imbalance in baseline features between the two treatment groups, or it may be the result of other events influencing kidney function that may have occurred after the patients' discharge from the hospital. 
On the basis of prior studies of the use of ultrafiltration in patients with acute decompensated heart failure, ${ }^{10,15,16}$ we anticipated that the patients in the ultrafiltration group would lose more weight than would those in the pharmacologic-therapy group. Ultrafiltration was discontinued early owing to multiple reasons other than attainment of a satisfactory fluid volume, a finding that shows the complexity of the use of ultrafiltration in patients with acute decompensated heart failure and the cardiorenal syndrome. Patients in the pharmacologic-therapy group had substantial diuresis as a result of the aggressive use of diuretics and adjuvant therapies.

The rates of death and rehospitalization did not differ significantly between the two treatment strategies, despite the increase in the creatinine level at 96 hours in the ultrafiltration group. Several retrospective studies have shown an association between worsening renal function and poor outcomes. ${ }^{17-19}$ Other trials, however, indicate that the relationships among the degree of clearing of congestion, changes in renal function, and outcomes are less clear. ${ }^{10,20-23}$ In the Diuretic Optimization Strategies Evaluation (DOSE) trial, worsening renal function in the high-dose furosemide group was not associated with worse outcomes. ${ }^{20}$ In the UNLOAD trial, a trend toward worsening renal function and greater weight loss in the ultrafiltration group was associated with a reduction in the rate of hospitalization for heart failure. ${ }^{10}$ The relationships among changes in renal function, degree of clearing of congestion, and outcomes in patients with acute decompensated heart failure are complex and require more study. In our trial, the rates of death or rehospitalization at 60 days were very high, showing the need for better therapies for this patient population.

Our study has several limitations. First, although the trial was randomized, the treatment assignments were not blinded, and biases on the part of study investigators may have affected the duration or relative intensity of ultrafiltration and pharmacologic therapy. Second, the safest and most effective rates of fluid removal, the duration of therapy, and the conditions for termination of ultrafiltration are unknown. A different intensity of ultrafiltration might have resulted in more fluid loss in the ultrafiltration group; however, the effect of a different intensity on renal function and outcomes is unknown. Finally, the results of the strategies tested here may not apply to other patient populations with acute decompensated heart failure, such as patients with less severe cardiorenal syndrome.

In summary, we conducted a randomized trial involving patients hospitalized for acute decompensated heart failure, worsened renal function, and persistent congestion. We found that the use of a stepped pharmacologic-therapy algorithm was superior to a strategy of ultrafiltration for the preservation of renal function, with the amount of weight loss at 96 hours similar with the two approaches. Ultrafiltration was associated with higher rates of adverse events.

\section{Supplementary Material}

Refer to Web version on PubMed Central for supplementary material.

\section{Acknowledgments}

The authors are solely responsible for the content of this article, which does not necessarily represent the official
views of the National Heart, Lung, and Blood Institute or the National Institutes of Health.

Supported by grants from the National Heart, Lung, and Blood Institute (U10HL084904 [for the data coordinating center], U10HL084861, U10HL084875, U10HL084877, U10HL084889, U10HL084890, U10HL084891, U10HL084899, U10HL084907, and U10HL084931 [for the clinical centers]).

We thank the patients who participated in this study, the Heart Failure Network site investigators and coordinators, the members of the Heart Failure Network data and safety monitoring board and protocol review committee, and 
the National Heart, Lung, and Blood Institute representatives — all of whom are listed in the Supplementary Appendix.

\section{References}

1. Ronco C, Cicoira M, McCullough PA. Cardiorenal syndrome type 1: pathophysiological crosstalk leading to combined heart and kidney dysfunction in the setting of acutely decompensated heart failure. J Am Coll Cardiol. 2012; 60:1031-42. [PubMed: 22840531]

2. Metra M, Davison B, Bettari L, et al. Is worsening renal function an ominous prognostic sign in patients with acute heart failure? The role of congestion and its interaction with renal function. Circ Heart Fail. 2012; 5:54-62. [PubMed: 22167320]

3. Hunt SA, Abraham WT, Chin MH, et al. Focused update incorporated into the ACC/AHA 2005 Guidelines for the Diagnosis and Management of Heart Failure in Adults: a report of the American College of Cardiology Foundation/American Heart Association Task Force on Practice Guidelines: developed in collaboration with the International Society for Heart and Lung Transplantation. Circulation. 2009; 119(14):e391-e479. Erratum, Circulation 2010;121(12)e258. [PubMed: 19324966]

4. Felker GM, Mentz RJ. Diuretics and ultrafiltration in acute decompensated heart failure. J Am Coll Cardiol. 2012; 59:2145-53. [PubMed: 22676934]

5. Freda BJ, Slawsky M, Mallidi J, Braden GL. Decongestive treatment of acute de-compensated heart failure: cardiorenal implications of ultrafiltration and diuretics. Am J Kidney Dis. 2011; 58:100517. [PubMed: 22014726]

6. Bart BA. Treatment of congestion in congestive heart failure: ultrafiltration is the only rational initial treatment of volume overload in decompensated heart failure. Circ Heart Fail. 2009; 2:499504. [PubMed: 19808381]

7. Bart BA, Goldsmith SR, Lee KL, et al. Cardiorenal rescue study in acute decompensated heart failure: rationale and design of CARRESS-HF, for the Heart Failure Clinical Research Network. J Card Fail. 2012; 18:176-82. [PubMed: 22385937]

8. Hotelling H. The generalization of Student's ratio. Ann Math Stat. 1931; 2:360-78.

9. Grant S, Aitchison T, Henderson E, et al. A comparison of the reproducibility and the sensitivity to change of visual analogue scales, Borg scales, and Likert scales in normal subjects during submaximal exercise. Chest. 1999; 116:1208-17. [PubMed: 10559077]

10. Costanzo MR, Guglin ME, Saltzberg MT, et al. Ultrafiltration versus intravenous diuretics for patients hospitalized for acute decompensated heart failure. J Am Coll Cardiol. 2007; 49:675-83. Erratum, J Am Coll Cardiol 2007;49:1136. [PubMed: 17291932]

11. Johnson, RA.; Wichern, DW. Applied multivariate statistical analysis. 6. Upper Saddle River, NJ: Pearson Prentice Hall; 2007. Multivariate linear regression models; p. 331-3.

12. Kaplan EL, Meier P. Nonparametric estimation from incomplete observations. J Am Stat Assoc. 1958; 53:457-81.

13. Cox DR. Regression models and life-tables. J R Stat Soc B. 1972; 34:187-220.

14. Marenzi G, Lauri G, Grazi M, Assanelli E, Campodonico J, Agostoni P. Circulatory response to fluid overload removal by extracorporeal ultrafiltration in refractory congestive heart failure. $\mathrm{J} \mathrm{Am}$ Coll Cardiol. 2001; 38:963-8. [PubMed: 11583865]

15. Bart BA, Boyle A, Bank AJ, et al. Ultra-filtration versus usual care for hospitalized patients with heart failure: the Relief for Acutely Fluid-Overloaded Patients with Decompensated Congestive Heart Failure (RAPID-CHF) trial. J Am Coll Cardiol. 2005; 46:2043-6. [PubMed: 16325039]

16. Bart BA, Walsh MM, Blake D, Goldsmith SR. Ultrafiltration for cardiorenal syndrome. J Card Fail. 2008; 14:531-3. [PubMed: 18672202]

17. Butler J, Forman DE, Abraham WT, et al. Relationship between heart failure treatment and development of worsening renal function among hospitalized patients. Am Heart J. 2004; 147:331-8. [PubMed: 14760333]

18. Forman DE, Butler J, Wang Y, et al. Incidence, predictors at admission, and impact of worsening renal function among patients hospitalized with heart failure. J Am Coll Cardiol. 2004; 43:61-7. [PubMed: 14715185] 
19. Owan TE, Hodge DO, Herges RM, Jacobsen SJ, Roger VL, Redfield MM. Secular trends in renal dysfunction and outcomes in hospitalized heart failure patients. J Card Fail. 2006; 12:257-62. [PubMed: 16679257]

20. Felker GM, Lee KL, Bull DA, et al. Diuretic strategies in patients with acute decompensated heart failure. N Engl J Med. 2011; 364:797-805. [PubMed: 21366472]

21. Testani JM, Chen J, McCauley BD, Kimmel SE, Shannon RP. Potential effects of aggressive decongestion during the treatment of decompensated heart failure on renal function and survival. Circulation. 2010; 122:265-72. [PubMed: 20606118]

22. O'Connor CM, Starling RC, Hernandez AF, et al. Effect of nesiritide in patients with acute decompensated heart failure. N Engl J Med. 2011; 365:32-43. Erratum, N Engl J Med 2011;365:773. [PubMed: 21732835]

23. Massie BM, O'Connor CM, Metra M, et al. Rolofylline, an adenosine $\mathrm{A}_{1}$-receptor antagonist, in acute heart failure. N Engl J Med. 2010; 363:1419-28. [PubMed: 20925544] 


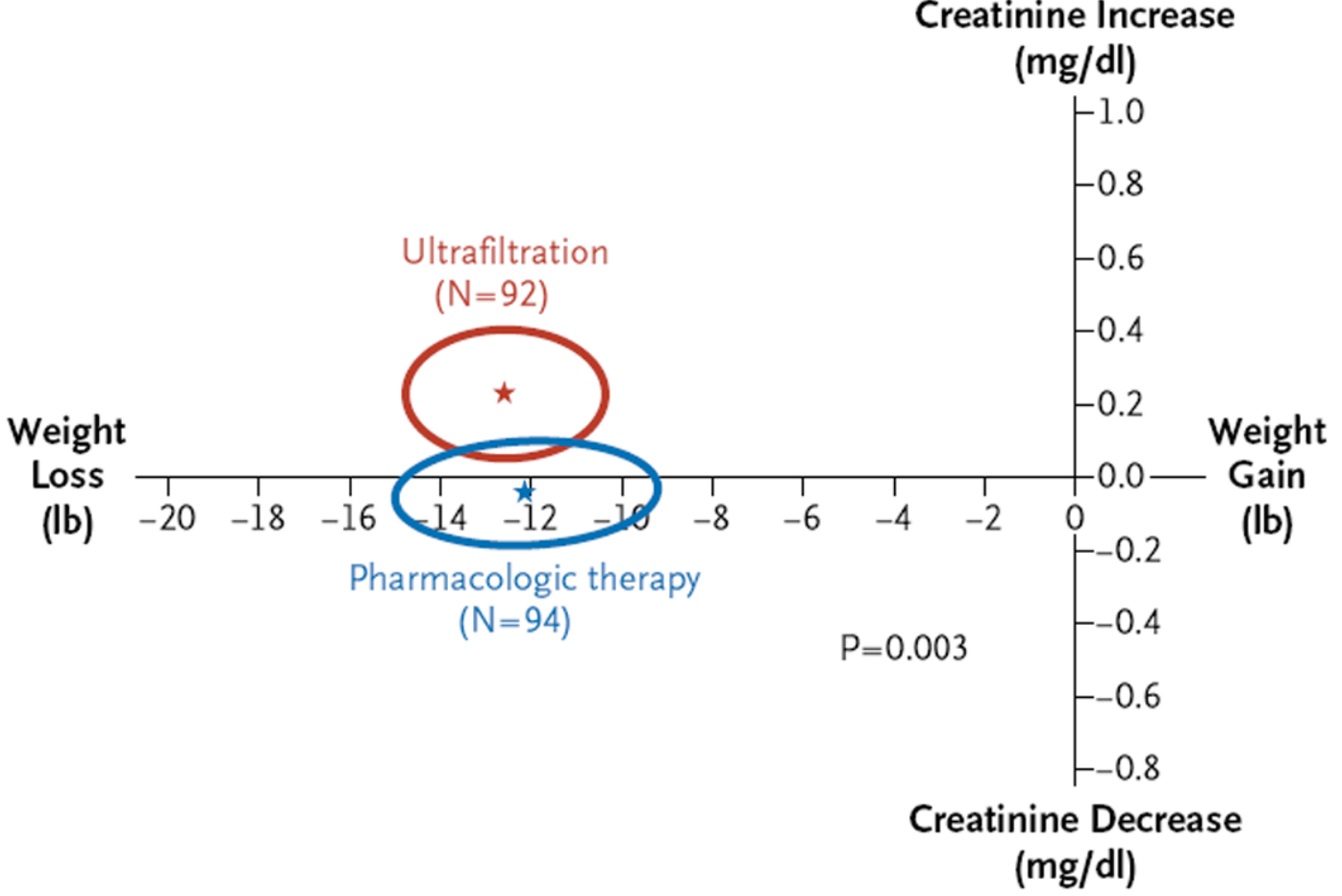

Figure 1. Changes in Serum Creatinine and Weight at 96 Hours (Bivariate Response)

The ellipses represent the $95 \%$ confidence regions and the stars the exact values for the mean changes in the serum creatinine level and weight at 96 hours in the ultrafiltration group and the pharmacologic-therapy group. Data from two patients who had been randomly assigned to the ultrafiltration group were excluded from the analysis: baseline creatinine measurements were missing for one patient, and all post-baseline creatinine measurements were missing for the other patient. To convert the values for creatinine to micromoles per liter, multiply by 88.4 . To convert the values for weight to kilograms, multiply by 0.45 . 
A Serum Creatinine

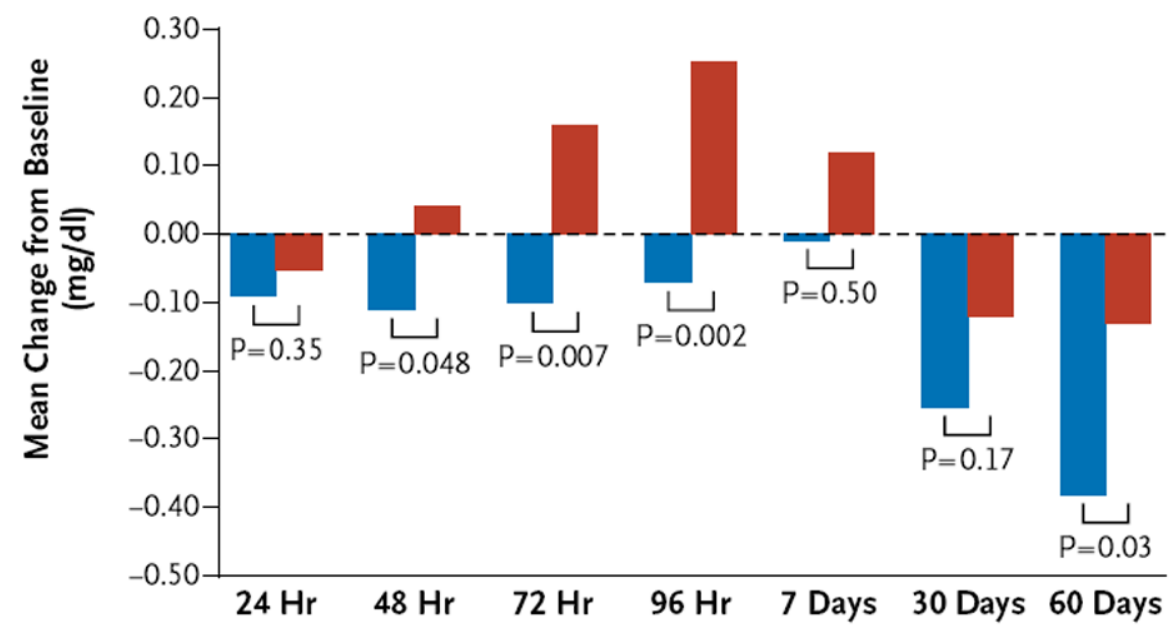

\section{B Body Weight}

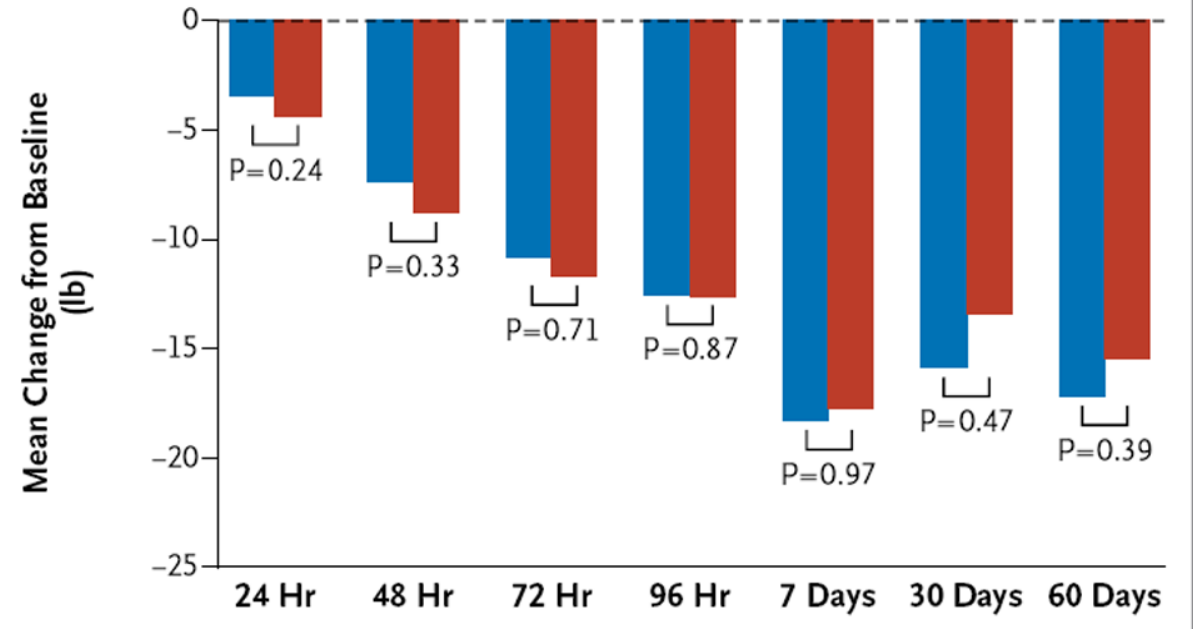

Figure 2. Changes from Baseline in Serum Creatinine and Body Weight at Various Time Points, According to Treatment Group

The $\mathrm{P}$ values were calculated with the use of a Wilcoxon test. The data on creatinine levels reflect results from testing in local laboratories only. 
Table 1

Baseline Characteristics of the Patients. *

\begin{tabular}{|c|c|c|}
\hline Characteristic & Pharmacologic Therapy $(N=94)$ & Ultrafiltration $(\mathrm{N}=94)$ \\
\hline \multicolumn{3}{|l|}{ Age $-\mathrm{yr}$} \\
\hline Median & 66 & 69 \\
\hline Interquartile range & $57-78$ & $61-78$ \\
\hline Male sex — no. $(\%)$ & $68(72)$ & $73(78)$ \\
\hline White race - no. $(\%)^{\dagger}$ & $67(71)$ & $72(77)$ \\
\hline \multicolumn{3}{|l|}{ Weight $-\mathrm{lb}$} \\
\hline Median & 234 & 207 \\
\hline Interquartile range & 190-292 & $172-265$ \\
\hline \multicolumn{3}{|l|}{ Ejection fraction $-\%$} \\
\hline Median & 35 & 30 \\
\hline Interquartile range & $25-55$ & $20-52$ \\
\hline Hospitalization for heart failure in previous $\mathrm{yr}$ - no./total no. (\%) & 73/92 (79) & $70 / 93(75)$ \\
\hline Ischemia as cause of heart failure - no. $(\%)$ & $48(51)$ & $66(70)$ \\
\hline History of atrial fibrillation or flutter - no. $(\%)$ & $48(51)$ & $54(57)$ \\
\hline Diabetes mellitus - no. $(\%)$ & $63(67)$ & $61(65)$ \\
\hline \multicolumn{3}{|l|}{ Medications received before hospitalization } \\
\hline ACE inhibitor or ARB — no. (\%) & $49(52)$ & $52(55)$ \\
\hline Beta-blocker - no. $(\%)$ & $73(78)$ & $74(79)$ \\
\hline Aldosterone antagonist - no. $(\%)$ & $17(18)$ & $21(22)$ \\
\hline \multicolumn{3}{|l|}{ Furosemide-equivalent diuretic } \\
\hline Patients receiving medication — no. $(\%)$ & $90(96)$ & $86(91)$ \\
\hline \multicolumn{3}{|l|}{ Furosemide-equivalent dose $-\mathrm{mg}$ /day } \\
\hline Median & 120 & 120 \\
\hline Interquartile range & $80-160$ & $80-240$ \\
\hline \multicolumn{3}{|l|}{ Blood urea nitrogen $-\mathrm{mg} / \mathrm{dl}$} \\
\hline Median & 50.5 & 48.7 \\
\hline Interquartile range & $39.0-64.0$ & $39.5-66.0$ \\
\hline \multicolumn{3}{|l|}{ Creatinine $-\mathrm{mg} / \mathrm{dl}^{*}$} \\
\hline Median & 2.09 & 1.90 \\
\hline Interquartile range & $1.71-2.65$ & $1.57-2.37$ \\
\hline \multicolumn{3}{|l|}{ Qualifying increase in creatinine $-\mathrm{mg} / \mathrm{dl}{ }^{\mathcal{S}}$} \\
\hline Median & 0.46 & 0.43 \\
\hline Interquartile range & $0.37-0.70$ & $0.35-0.60$ \\
\hline \multicolumn{3}{|l|}{ NT-proBNP $-\mathrm{pg} / \mathrm{ml}$ I/ } \\
\hline Median & 4007 & 5013 \\
\hline Interquartile range & $1128-8534$ & $2310-10381$ \\
\hline
\end{tabular}


There were no significant differences between the groups in the baseline characteristics listed here, with the exception of ischemia as the cause of heart failure $(\mathrm{P}=0.007)$. ACE denotes angiotensin-converting enzyme, ARB angiotensin-receptor blocker, and NT-proBNP N-terminal pro-brain natriuretic peptide. To convert the values for creatinine to micro-moles per liter, multiply by 88.4 . To convert the values for blood urea nitrogen to micromoles per liter, multiply by 0.357 . To convert the values for weight to kilograms, multiply by 0.45 .

Race was self-reported.

${ }^{*}$ The creatinine values reflect the results of testing performed at the core laboratory only.

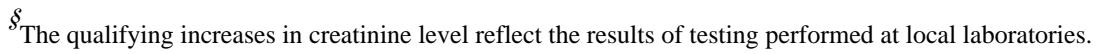

${ }^{I}$ The reference range for NT-proBNP is 5 to $70,000 \mathrm{pg} / \mathrm{ml}$. 
Table 2

Secondary End Points. ${ }^{*}$

\begin{tabular}{|c|c|c|c|}
\hline End Point & $\begin{array}{l}\text { Pharmacologic } \\
\text { Therapy }(\mathrm{N}=94)\end{array}$ & $\begin{array}{l}\text { Ultrafiltration }(\mathbf{N}= \\
\text { 94) }\end{array}$ & P Value \\
\hline \multicolumn{4}{|l|}{ Significant body weight loss and renal improvement - no. $(\%)^{\dagger}$} \\
\hline At $96 \mathrm{hr}$ & $20(21)$ & $16(17)$ & 0.62 \\
\hline At 7 days & $20(21)$ & $15(16)$ & 0.52 \\
\hline Worsening condition or crossover during the first 7 days - no./total no. $(\%)^{*}$ & $17 / 94(18)$ & 21/93 (23) & 0.45 \\
\hline Clinical decongestion at $96 \mathrm{hr}-$ no./total no. $(\%)^{\mathcal{S}}$ & $7 / 80(9)$ & $8 / 82(10)$ & 0.83 \\
\hline Change in sodium from baseline to $96 \mathrm{hr}-\mathrm{mmol} / \mathrm{liter}$ & $0.0 \pm 3.6$ & $-2.3 \pm 3.5$ & $<0.001$ \\
\hline Change in hemoglobin from baseline to $96 \mathrm{hr}-\mathrm{g} / \mathrm{dl}$ & $0.38 \pm 0.76$ & $-0.01 \pm 0.92$ & 0.002 \\
\hline Change in NT-proBNP from baseline to $96 \mathrm{hr}-\mathrm{pg} / \mathrm{ml}$ & $-979 \pm 2902$ & $-814 \pm 9239$ & 0.30 \\
\hline Change in cystatin $\mathrm{C}$ from baseline to $96 \mathrm{hr}-\mathrm{mg} /$ liter & $0.14 \pm 0.52$ & $0.22 \pm 0.52$ & 0.37 \\
\hline Change in blood urea nitrogen from baseline to $96 \mathrm{hr}-\mathrm{mg} / \mathrm{dl}$ & $5.68 \pm 18.29$ & $12.54 \pm 24.81$ & 0.02 \\
\hline Change in glomerular filtration rate from baseline to $96 \mathrm{hr}-\mathrm{ml} / \mathrm{min} / 1.73 \mathrm{~m}^{2}$ & $1.67 \pm 10.94$ & $0.93 \pm 14.60$ & 0.66 \\
\hline Change in score on global well-being scale from baseline to $96 \mathrm{hr} / \mathrm{I}$ & $22.8 \pm 25.8$ & $13.7 \pm 27.9$ & 0.33 \\
\hline Change in score on dyspnea assessment scale from baseline to $96 \mathrm{hr} / \mathrm{l}$ & $20.5 \pm 27.8$ & $16.5 \pm 29.2$ & 0.57 \\
\hline Total net fluid loss from randomization to $96 \mathrm{hr}-\mathrm{ml}$ & $7082 \pm 4183$ & $7443 \pm 4329$ & 0.59 \\
\hline $\begin{array}{l}\text { Change in furosemide-equivalent dose from preadmission to discharge }-\mathrm{mg} \text { / } \\
\text { day }\end{array}$ & $2.2 \pm 166.5$ & $-20.6 \pm 116.0$ & 0.18 \\
\hline Death - no. $(\%)$ & $13(14)$ & $16(17)$ & 0.55 \\
\hline \multicolumn{4}{|l|}{ Hospitalization - no./total no. $(\%)$} \\
\hline For heart failure & $24 / 93(26)$ & $23 / 90(26)$ & 0.97 \\
\hline For any cause & $37 / 93(40)$ & $46 / 90(51)$ & 0.12 \\
\hline Unscheduled emergency department or clinic visit - no./total no. (\%) & $13 / 93(14)$ & $19 / 90(21)$ & 0.21 \\
\hline
\end{tabular}

Plus-minus values are means \pm SD.

Significant weight loss was defined as a loss of $3 \mathrm{~kg}(6.6 \mathrm{lb})$ or more; significant renal improvement was defined as a decrease in the creatinine level of $0.3 \mathrm{~g}$ per deciliter ( $27 \mu \mathrm{mol}$ per liter) or more.

${ }^{*}$ Worsening condition was defined as death, worsening or persistent heart failure, need for dialysis, or the occurrence of a serious adverse event.

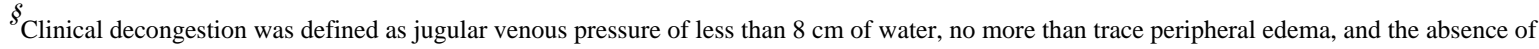
orthopnea.

II Global well-being and dyspnea were assessed with the use of a visual-analogue scale that ranged from 0 to 100 , with higher scores indicating greater well-being and lesser severity of dyspnea, respectively. 9 
Table 3

Serious Adverse Events.

\begin{tabular}{|lcc|}
\hline Event & Pharmacologic Therapy (N = 94) & Ultrafiltration (N = 94) \\
\hline \multicolumn{2}{|c|}{ no. of patients (\%) } \\
\hline Any & $54(57)$ & $68(72)$ \\
\hline Heart failure & $28(30)$ & $31(33)$ \\
\hline Other cardiovascular disorder & $5(5)$ & $6(6)$ \\
\hline Renal failure & $14(15)$ & $17(18)$ \\
\hline Anemia or thrombocytopenia & $5(5)$ & $8(9)$ \\
\hline Catheter-site hemorrhage & 0 & $2(2)$ \\
\hline Electrolyte disorder ${ }^{*}$ & $3(3)$ & 0 \\
\hline Gastrointestinal hemorrhage & $3(3)$ & $7(7)$ \\
\hline Pneumonia or other respiratory disorder & $6(6)$ & $10(11)$ \\
\hline Sepsis, bacteremia, or cellulitis & $4(4)$ & $8(9)$ \\
\hline Other & $19(20)$ & $17(18)$ \\
\hline
\end{tabular}

Included in this category are hyperkalemia, hypokalemia, hypernatremia, hypoatremia, and hyperuricemia. 